\title{
Sonochemistry: from Basic Principles to Innovative Applications
}

\author{
${\text { Gregory } \text { Chatel }^{1} \cdot \text { Juan Carlos Colmenares }}^{2}$
}

Published online: 2 January 2017

(C) Springer International Publishing Switzerland 2016

The term "sonochemistry" is used to describe the chemical and physical processes occurring in solution through energy provided by ultrasound (in the range from $20 \mathrm{kHz}$ to $2 \mathrm{MHz}$ ). The effects of ultrasound are the consequence of the cavitation phenomenon, namely the formation, growth, and collapse of gaseous microbubbles in liquid phase. The intense local effects (mechanical, thermal, and chemical) due to the sudden collapse of these micrometric bubbles lie at the origin of all applications of sonochemistry. Use of ultrasound in chemical processes increased between 1980 and 2000, and the corresponding literature has increased enormously in volume from 2000 onwards, reporting many applications in several areas including but not limited to sonocatalysis, organic chemistry, materials preparation, polymer chemistry, biomass conversion, extraction, electrochemistry, enzymatic catalysis, and environmental remediation.

The potential of sonochemistry is often directly connected to the choice of the sonochemical parameters and experimental conditions. The first contribution to this topical collection, provided by Prof. Ashokkumar's research group, provides theoretical considerations regarding the use of ultrasound in the laboratory, to better

This article is part of the Topical Collection "Sonochemistry: From basic principles to innovative applications" edited by Juan Carlos Colmenares Q. and Gregory Chatel.

Gregory Chatel

gregory.chatel@univ-smb.fr;

http://gregorychatel-chemistry.com/

$\bowtie$ Juan Carlos Colmenares

jcarloscolmenares@ichf.edu.pl;

http://photo-catalysis.org

1 Laboratoire de Chimie Moléculaire et Environnement (LCME), UFR Sciences et Montagne (SceM), Université Savoie Mont Blanc, 73376 Le Bourget Du Lac Cedex, France

2 Institute of Physical Chemistry of the Polish Academy of Sciences (PAS), Kasprzaka 44/52, 01-224 Warsaw, Poland 
understand the mechanisms involved and determine which parameters can affect the observed results. Specifically, a detailed discussion on single-bubble sonochemistry is provided in this contribution.

Further contributions highlight how sonochemistry represents a key area for research and innovation in chemistry. Indeed, the conditions obtained in a medium submitted to ultrasound can account for a large number of physicochemical effects, such as enhanced kinetics of chemical reactions, changes in reaction mechanisms, emulsification effects, erosion, crystallization, and precipitation, among others. Sonochemistry is multidisciplinary, and recent advances highlighting this are reported in this topical collection, in particular applications such as organic chemistry (Prof. Draye's group), synthesis of nanostructured materials (Prof. Suslick), synthesis of photoactive materials (Prof. Colmenares' group), advanced oxidation processes (Prof. Neppolian's group), and treatment of sewage sludge (Prof. Zielewicz). Results in these areas show that the improvements achieved require use of recently developed sonochemical processes.

The combination of sonochemistry with other innovative technologies is also highlighted, using microwave irradiation (Prof. Cravotto's group), ionic liquids as solvent or catalyst (Dr. Chatel), and coupling with microfluidics (Prof. Rivas' group). In all these cases, synergistic effects are observed, representing a new source of innovation for further research.

The final contribution, provided by Prof. Gogate's group, covers laboratory equipment and the opportunity to scale up the technology for maximum process intensification benefits. We conclude by stating that scientific rigor is essential in the sonochemistry area to understand the associated mechanisms and benefit from the full potential offered by ultrasound.

The editors kindly acknowledge the Topics in Current Chemistry editorial office (Springer) for inviting us to compile this comprehensive topical collection on sonochemistry. We would also like to extend our most sincere gratitude to international experts for their time and consideration regarding this project, as well as for their general contributions to sonochemistry science; It was pleasing and instructive to work with them on this topical collection. Last but not least, we sincerely thank our publishing editor, Elizabeth Hawkins, and assistant editor, Na $\mathrm{Xu}$, who patiently and kindly took us through the development of this topical collection over the past few months to achieve this impressive final result, which would not have been possible without such support.

We sincerely hope that this topical collection will be useful both to (bio)chemistry, chemical engineering, and materials science students, as well as to graduates interested in green chemical technologies, and wish you an enjoyable and satisfactory read. 


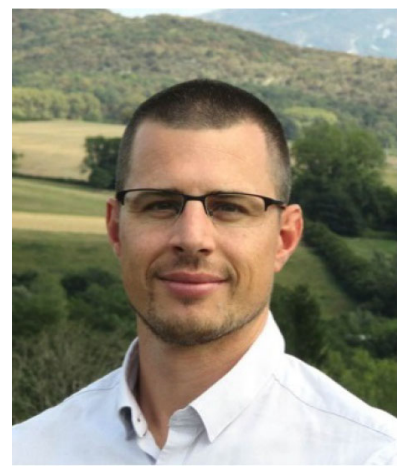

Dr. Gregory Chatel received his Ph.D. degree in 2012 from the Universite de Grenoble (France) under the supervision of Prof. M. Draye and Prof. B. Andrioletti, studying the fundamentals of and developing a sonochemical method involving ionic liquids for epoxidation of various alkenes. In 2013, he joined Prof. R. D. Rogers' group at the Center for Green Manufacturing at The University of Alabama (USA) as a postdoctoral research fellow. His research has focused on applications of ionic liquids in green chemistry, separation, and biomass processing. At the end of 2013, Dr. Chatel joined the Institut de Chimie des Milieux et Matériaux de Poitiers (IC2MP) of the Université de Poitiers (France) as an assistant professor to develop a biomass valorization program based on nonconventional media/techniques, in particular via sonochemistry. In 2014, he became the first president of the French national Young Chemists' Network (RJ-SCF) of the French Chemical Society. In 2016, he joined the Laboratoire de Chimie Moléculaire et Environnement (LCME) of the Université Savoie Mont Blanc (France) to develop his work in organic sonochemistry through new original processes and applications.
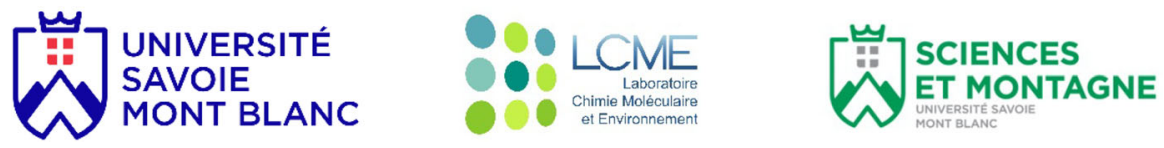


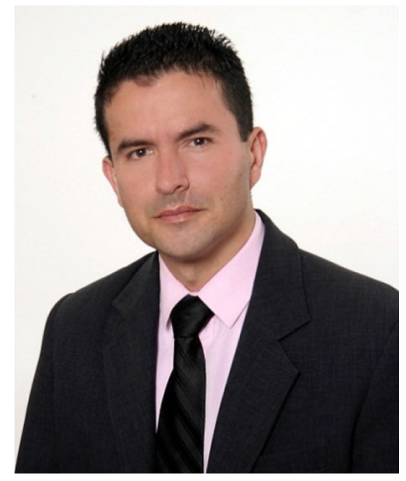

Prof. Dr. Juan Carlos Colmenares Q. graduated from Warsaw University of Technology (Chem. Eng. 1995) and obtained his M.Sc. (1997) in catalysis for organic technology and Ph.D. (2004) in chemical and material sciences from the same university, and his scientific degree of habilitation (D.Sc. 2015) from the Institute of Physical Chemistry of the Polish Academy of Sciences in Poland. His interests range from materials science, nanotechnology, and heterogeneous catalysis to biomass/CO valorization, solar chemicals, sonication, photocatalysis, and water/air purification. After obtaining his Ph.D., he worked at the University of Córdoba, Spain (2005-2006) in Prof. Marinas' group as a postdoctoral fellow and at the University of Southern California, Los Angeles (USA) (2006-2009) in Prof. G. A. Olah's (Nobel Prize in Chemistry) group as a postdoctoral research associate. He is a Marie Skłodowska-Curie fellow. He serves as an expert evaluator for many important scientific journals/institutions and chemical companies (Colombia, USA, Poland, Spain), and as a member of the editorial advisory board of the Sustainable Chemical Processes journal (Chemistry Central-Springer). He has coauthored more than 60 works published in international scientific journals and books, and filed three patent applications. Presently, he is working as an associate professor at the Institute of Physical Chemistry of the Polish Academy of Sciences in Poland.
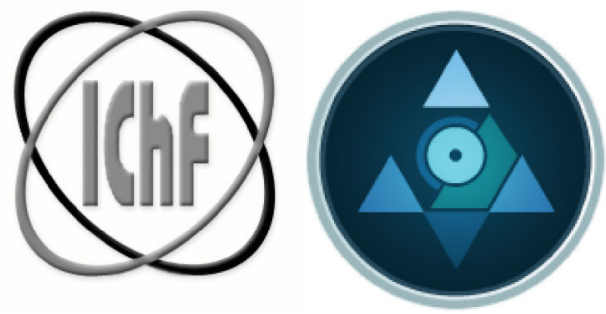